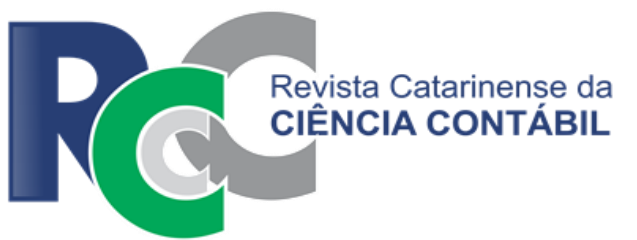

\title{
OS EFEITOS DOS INTANGÍVEIS NAS PREVISÕES DOS ANALISTAS FINANCEIROS
}

\author{
EFFECTS OF INTANGIBLES ON FINANCIAL ANALYSTS' FORECAST
}

\author{
GILMAR GOMES GAZZONI JUNIOR \\ Universidade Federal do Espírito Santo. Endereço: Av. Fernando Ferrari, 514 \\ | Goiabeiras | 29075-910 | Espírito Santo/ES | Brasil. \\ (D)http://orcid.org/0000-0003-0396-8386 \\ junior_gazoni@hotmail.com
}

\section{JOÃO JOSÉ FERREIRA SIMÕES}

Universidade Federal de Minas Gerais. Endereço: Av. Antônio Carlos, 6627| Pampulha | 31270-010 | Belo Horizonte/MG | Brasil.

(D)http://orcid.org/0000-0002-0095-0458

simoes.joao@yahoo.com.br

\author{
MARCELO MOLL BRANDÃO \\ Universidade Federal do Espírito Santo. Endereço: Av. Fernando Ferrari, 514 \\ | Goiabeiras | 29075-910 | Espírito Santo/ES | Brasil. \\ Dhttp://orcid.org/0000-0002-8593-734X \\ mollmkt@gmail.com
}

\author{
ANTONIO ARTUR DE SOUZA \\ Universidade Federal de Minas Gerais. Endereço: Av. Antônio Carlos, 6627| \\ Pampulha | 31270-010 | Belo Horizonte/MG | Brasil. \\ (D)http://orcid.org/0000-0002-4725-0758 \\ artur@face.ufmg.br
}

\section{RESUMO}

Este estudo objetivou verificar a influência dos intangíveis sobre a acurácia e a dispersão das previsões de lucros feita pelos analistas financeiros. A execução deste estudo foi motivada ao observar que a literatura sugere que os intangíveis podem influenciar as previsões dos analistas. Contudo, os resultados verificados são divergentes, não havendo um consenso a respeito da direção dessa influência nos trabalhos à questão. Para atingir os objetivos de pesquisa, utilizou-se uma amostra de empresas americanas não financeiras com ações negociadas na Nasdaq, no período de 1995 a 2016, a partir do método OLS (Ordinary Least Squares - Mínimos Quadrados Ordinários), conforme adotado pela maior parte da literatura internacional encontrada acerca do tema. A escolha dessa amostra se deu pela maior disponibilidade dos dados dos intangíveis e da cobertura dos analistas, pela comparabilidade com resultados existentes na literatura e pelo fato de a respectiva bolsa concentrar empresas com maior grau de intangibilidade, foco do trabalho. Desse modo, os principais resultados apontaram que os investimentos em P\&D e ativos intangíveis reconhecidos são capazes de melhorar as previsões dos analistas. Contudo, o Goodwill mostrouse negativamente relacionado com as previsões dos analistas ao reduzir a acurácia e aumentar a dispersão das previsões. Concluiu-se, então, a possibilidade em confirmar a existência de 
associação entre os intangíveis e as previsões dos analistas, além de ter fornecido indícios de que a direção dessa influência não pode ser generalizada a todos intangíveis, a depender do nível de incerteza e complexidade informacional do intangível estudado.

Palavras-chave: Intangível. Previsões dos analistas. Acurácia. Pesquisa e Desenvolvimento, Goodwill.

\begin{abstract}
The purpose of this research was to verify the influence of intangibles on the accuracy and dispersion of profit forecasts made by financial analysts. This study was motivated by observing that the literature suggests that intangibles can influence analysts' forecasts; however, the results are divergent, and there is no consensus regarding the direction of this influence in the studies found that address this theme. To achieve the research objectives, a sample of American nonfinancial companies with shares traded on Nasdaq from 1995 to 2016 was used from the OLS (Ordinary Least Squares) method, as adopted by most of the literature on the subject. The choice of such sample was due to the greater availability of intangible data and analysts' coverage, comparability with results of previous research in the literature and also because the respective stock exchange concentrates companies with greater degree of intangibility. The main results indicated that investments in $R \& D$ and recognized intangible assets were capable of improving analysts' forecasts. However, Goodwill was negatively correlated with analysts' forecasts by reducing accuracy and widening forecast dispersion. The evidence suggested an association between intangible and analysts' forecasts, providing evidence that the direction of this influence cannot be generalized to all intangibles, depending on the level of uncertainty and information complexity of the intangible studied.
\end{abstract}

Keywords: Intangible. Analysts'forecast. Accuracy. Research \& Development. Goodwill.

\title{
1 INTRODUÇÃO
}

A economia mundial está em constante transformação, evoluindo e trazendo novas formas de negócios e produtos. Grande parte deste processo de evolução dos mercados é impulsionada pela concorrência existente entre as empresas, intensificada pelo processo de globalização. Jia (2017) destaca que a concorrência tem evoluído cada vez mais em torno do capital intelectual das empresas, tornando-se a competência de maior importância. Neste contexto, as empresas buscam meios para se diferenciar de seus concorrentes e alcançar melhores resultados, investindo em pesquisa e desenvolvimento $(\mathrm{P} \& \mathrm{D})$ e visando alcançar melhores resultados com o desenvolver de processos e produtos inovadores (McDermott, 1999).

Esta relação entre a inovação e a obtenção de melhor desempenho já foi abordada pela literatura. Perez e Famá (2006), assim, analisaram a relação entre a inovação e o desempenho empresarial por meio dos ativos intangíveis não reconhecidos pelas demonstrações contábeis. Os resultados observados apontam que as empresas intangível-intensivas apresentam desempenho significativamente superior. Simões e Louzada (2016), por sua vez, demonstraram empiricamente a relação entre os ativos intangíveis e a vantagem competitiva, evidenciando que a persistência nos níveis de intangível apresentam impacto positivo na geração do ROIC.

Sabendo da importância dos intangíveis para o desempenho empresarial, os analistas financeiros devem observar tal aspecto em suas previsões de lucro. Segundo Cummins (2005), cabe aos analistas financeiros refletir o valor dos intangíveis em suas previsões, de forma a avaliar o potencial de geração de lucros adicionais que podem contribuir materialmente para o resultado final da empresa. Dessa forma, trabalhos anteriores destacam que a maior divulgação de informações relativas aos ativos intangíveis por parte das empresas ajuda a reduzir a assimetria 
informacional, aumentar a liquidez das ações, atrair investidores, aumentar a cobertura e, consequentemente, contribuir para a melhora das previsões (Diamond \& Verrecchia, 1991; Lang \& Lundholm, 1996; Srinivasan, 2007). Destaca-se, com isso, a discricionariedade envolvida na decisão de reconhecimento dos ativos intangíveis, a qual permite que os administradores sinalizem ao mercado suas perspectivas (Dinh, Eierle, Schultze \& Steeger, 2015; Matolcsy \& Wyatt, 2006).

Outros trabalhos apontam que, em razão do maior grau de complexidade e incerteza dos ativos intangíveis, ações como adequado reconhecimento, a mensuração e a avaliação são dificultadas e, consequentemente, afetam as previsões. Gu e Wang (2005) nesta esteira, apresentam que, nas últimas décadas, o aumento de tamanho dos intangíveis, em proporção e contribuição para os resultados, tornou-se um "problema" para os analistas financeiros, pois parte dos ativos intangíveis que não são reconhecidos pela contabilidade nas demonstrações financeiras. $\mathrm{E}$, quando reconhecidos, grande parte dos investimentos em intangíveis não são devidamente identificados nas demonstrações financeiras pela sua dificuldade de separação de outros gastos meramente operacionais (Lev, 2005). Além disso, os investimentos em P\&D tendem a ser exclusivos e privados à empresa investidora, o que limita a comparação entre empresas no processo de obtenção de informações para tomada de decisões (Palmon \& Yezegel, 2012). Ou seja, a incerteza de benefícios econômicos futuros atrelada aos ativos intangíveis aumenta a dificuldade de os analistas terem uma compreensão adequada da capacidade das empresas em gerar fluxo de caixa futuro, culminando em uma maior dificuldade na percepção a longo prazo da empresa.

A partir dos estudos anteriores observa-se que, apesar de haver reconhecimento da existência de relação entre os intangíveis e as previsões dos analistas, ainda não há um consenso sobre a direção desta influência. De um lado, aponta-se que os intangíveis fornecem informações úteis acerca da expectativa de lucros futuros. Por outro lado, defende-se que a incerteza e complexidade desta informação pode dificultar a adequada avaliação do futuro fluxo de caixa esperado pelos analistas. Motiva-se, assim, a realização de um trabalho que vise ao preenchimento da lacuna existente ao contribuir com o debate e também ao entendimento da existência de distintos resultados em busca de um consenso sobre o tema. Diante do exposto, pretende-se responder o seguinte problema de pesquisa: Qual a relação entre as previsões de lucro dos analistas financeiros e os diferentes tipos de intangíveis apresentados nas demonstrações contábeis?

Para responder ao questionamento da pesquisa, traçou-se como objetivo geral verificar a influência dos intangíveis sobre a acurácia e a dispersão das previsões de lucros feita pelos analistas financeiros. Especificamente, busca-se analisar se (i) os intangíveis reconhecidos nas demonstrações financeiras, (ii) os investimentos em P\&D e (iii) o Goodwill exerceram influência sobre a acurácia e a dispersão das previsões dos analistas.

Ante o exposto, justifica-se o desenvolvimento desta pesquisa, em virtude, primeiramente, do crescimento da importância dos intangíveis aos longos dos anos. Segundo Santos, Gomes, Fernandes, Pinheiro e Schmidt (2006), a geração de valor destes ativos tem aumentado significativamente, haja vista o crescimento da materialidade de seus valores na composição do ativo total das empresas. Outro ponto que evidencia o crescimento dos intangíveis é o índice de valor de mercado em relação ao valor contábil das empresas listadas na S\&P 500, que subiu de um, no início da década de 1980, para seis, no ano de 2001 (Lev, 2001). Os estudos sobre intangíveis ganharam importância, assim, diante de sua complexidade informacional e de avaliação (Penman, 2009). Jia (2017), então, destaca duas motivações para este tipo de estudo: (i) a inovação, que se tornou um elemento fundamental para o sucesso e crescimento da empresa a longo prazo e (ii) as implicações dos investimentos em intangíveis sobre os lucros futuros, que são complexas e de difícil avaliação (Ali, Ciftci, \& Cready, 2012). Além disso, é de suma importância entender como tal fator pode influenciar o analista diante do seu importante papel que, segundo Luo, Homburg e Wieseke (2010), gera valor aos investidores de duas formas: (a) analisando informações publicamente disponíveis com maior habilidade e (ii) coletando informações indisponíveis ao público em geral, importantes à adequada avaliação da empresa. 
Dito isso, esta pesquisa pretende contribuir com a discussão ao, diferentemente de estudos anteriores - além de utilizar proxies já utilizadas para investigar os ativos intangíveis, como o investimento em P\&D e os ativos intangíveis reconhecidos nas demonstrações financeiras -, preencher uma lacuna existente na discussão, adicionando o intangível de Goodwill. Além disso, buscou-se analisar, separadamente, a influência sobre as previsões dos analistas financeiros (i) dos ativos intangíveis reconhecidos nas demonstrações financeiras, (ii) dos investimentos em P\&D e (iii) do Goodwill, já que esta pode ser distinta entre os tipos de intangíveis em razão do grau de complexidade e incerteza de cada um, podendo ajudar a entender a existência dos distintos resultados existentes até o momento.

\section{REFERENCIAL TEÓRICO}

\subsection{Ativo e ativos intangíveis}

Os dois principais atributos de um ativo, conforme Santos et al. (2006), são o controle dos recursos e a capacidade de proporcionar benefícios econômicos futuros. $\mathrm{O}$ ativo é um recurso controlado pela entidade, sobre o qual há expectativa de benefícios econômicos futuros. Caso não exista tal expectativa, direta ou indiretamente, não há um ativo. Conforme Lev (2005), o ativo intangível, como qualquer ativo, também é fonte de benefícios futuros, mas não possui encarnação física. Lev (2001) define um ativo intangível como um direito a benefícios futuros que não possui corpo físico ou financeiro. Entretanto, na Contabilidade, na Economia e na Administração, os ativos intangíveis recebem diferentes denominações, como intangível, capital conhecimento e capital intelectual. Segundo Zanoteli (2015, p. 143), o significado, em essência, é o mesmo: "direito não físico sobre benefícios futuros".

Lev (2005) categoriza os ativos intangíveis como segue: (i) Produtos/Serviços: através do conhecimento obtido pelo investimento em P\&D que pode surgir futuramente, marcas e/ou patentes que possibilitam às empresas a oferta de novos produtos ou serviços aos seus clientes; (ii) Relação com clientes: está associada à lealdade destes, o que permite o aumento dos preços ou garante uma grande participação no mercado; (iii) Recursos humanos: configurados práticas únicas adotadas pela empresa, as quais aumentam consideravelmente a produtividade do trabalho; (iv) Capital organizacional: surge através de projetos únicos e processos de negócios, os quais permitem às empresas superarem os concorrentes por geração de maiores receitas ou redução de custos de produção.

Segundo Lev (2001), o investimento em P\&D é o foco dos pesquisadores por ser divulgado nas demonstrações de resultado separadamente de outros intangíveis. Em contrapartida, na perspectiva de Damodaran (2007), o Goodwill é o ativo intangível mais reconhecido nas demonstrações contábeis. O Goodwill representa a parte do valor pago referente à expectativa de rentabilidade futura de um ativo intangível, também composto pelo valor de mercado e valor contábil (Martins, Diniz \& Miranda, 2012). Para Iudicibus (2010), o Goodwill constitui-se pelo excesso do custo na aquisição de uma empresa, em função da sua expectativa de rentabilidade futura sobre seus ativos e passivos assumidos.

Contudo, o reconhecimento, a mensuração e, consequentemente, a avaliação dos intangíveis são complexos e fonte de ampla discussão pela Contabilidade por diversos fatores, dentre os quais é possível destacar que: (I) para a segregação entre gastos operacionais e investimentos, em intangíveis, seriam necessários muitos julgamentos subjetivos; (II) nem todos os investimentos em intangíveis geram retorno futuro; (III) há dificuldade em mensurá-los (Kanodia, Sapra \& Venugopalan, 2004). Isso ocorre, conforme Scott (2012), dado a maior parte dos ativos intangíveis serem gerados internamente com seus custos sendo diluídos ao longo dos anos.

Dessa forma, o fato de nem todos os ativos intangíveis serem reportados nas demonstrações financeiras torna-se o maior entrave no estudo da relação dos intangíveis e o desempenho da 
empresa (Lev, 2001). Isto é, apenas parte dos intangíveis, cuja mensuração e reconhecimento podem ser realizados adequadamente, podem ser reconhecidos como ativos intangíveis no balanço patrimonial, tornando o ativo intangível reconhecido no balanço como o tipo de intangível com menor grau de incerteza. O P\&D, apesar de fornecer importantes informações sobre o futuro da entidade, apresenta uma maior incerteza sobre os benefícios futuros que podem ser gerados, e o Goodwill representa uma parte de um montante pago cuja mensuração dificilmente é disponibilizada aos usuários externos para uma adequada análise pelos analistas financeiros.

\subsection{Acurácia dos analistas e os ativos intangíveis}

Os intangíveis são considerados características únicas das empresas, os quais permitem que estas se diferenciem de seus concorrentes. Os investimentos em ativos intangíveis, como P\&D (Pesquisa e Desenvolvimento), são essenciais para ganhar vantagem competitiva e, consequentemente, proporcionar aumento do lucro corporativo (McDermott, 1999; Perez \& Famá, 2006). Prova disso, Currim, Lim e Zhang (2018) evidenciaram que, mesmo em períodos de pressão, empresas que conseguem manter seus investimentos em $\mathrm{P} \& \mathrm{D}$ são recompensadas, apresentando um melhor desempenho a longo prazo.

Sabendo disso, Cummins (2005) ressalta que os analistas devem refletir o valor dos ativos intangíveis em suas previsões caso avaliem que estes podem gerar lucros adicionais e contribuir materialmente para o resultado final da empresa num período de cinco anos. Analisando isso, Abeysekera (2016), a partir de experimentos com analistas, sugere que tanto as marcas desenvolvidas internamente quanto as marcas compradas são consideradas pelos analistas e tratadas de forma equivalente para efeito das previsões de lucros nos relatórios anuais. Neste processo, os analistas desempenham um importante papel, mitigando a assimetria informacional, levando informações aos investidores, analisando informações públicas com maior habilidade e coletando informações não disponíveis ao público em geral, auxiliando, com isso, na tomada de decisões (Abhayawansa \& Guthrie, 2016; Luo et al., 2010).

Diante disso, Kwon (2002) evidenciou que as empresas que utilizam mais tecnologia em seus processos - empresas com maior grau de intangibilidade - atraem maior cobertura dos analistas financeiros devido à maior expectativa de crescimento. $\mathrm{O}$ autor explica que, quanto maior o número de analistas seguindo determinada empresa, maior será a acessibilidade de informações, o que, por sua vez, levaria a melhores previsões. Analisando tal relação, Bae, Hur, Lee e Goh (2017) analisam a influência das citações de patentes sobre as previsões dos analistas financeiros, de modo que seus achados indicam a maior chance de os analistas realizarem previsões de longo prazo para empresas com mais citações de patentes. Ou seja, empresas com maior grau de intangibilidade atraem maior número de analistas em virtude do seu maior potencial de ganho, gerando um aumento de informações analisadas com maior habilidade pelos analistas, permitindo uma melhor avaliação e previsão destes. Destaca-se, também, que a discricionariedade envolvida na decisão de reconhecimento dos ativos intangíveis permite que os administradores sinalizem ao mercado suas perspectivas e, quanto maior a quantidade de sinalizações, maior será a quantidade de informações disponíveis aos analistas (Dinh et al., 2015).

$\mathrm{Na}$ mesma perspectiva, Matolcsy e Wyatt (2006) encontraram evidências de que, em média, o reconhecimento do intangível nas demonstrações financeiras associa-se a lucros mais previsíveis, sendo capaz de fornecer maiores informações a respeito do desempenho futuro da empresa e, assim, auxiliar na previsibilidade dos lucros futuros. Dessa forma, a maior divulgação de informações relacionadas aos benefícios futuros gerados pelos ativos intangíveis reduziria a assimetria informacional, aumentando a liquidez das ações, atraindo investidores, aumentando a cobertura e, consequentemente, melhorando as previsões dos analistas (Diamond \& Verrecchia, 1991; Lang \& Lundholm, 1996; Srinivasan, 2007). Em outro trabalho, Maaloul, Ben Amar e Zeghal (2016) apresentam a relação entre o disclosure voluntário dos intangíveis e as previsões de ganhos dos analistas financeiros. Em seus achados, as evidências apontam que o aumento do 
disclosure dos intangíveis impacta a acurácia e a dispersão das previsões de lucros dos analistas, além das recomendações favoráveis de consenso deles.

Contudo, outros aspectos inerentes aos ativos intangíveis tornam desafiadora a mensuração da expectativa dos benefícios econômicos futuros, podendo dificultar a previsibilidade dos lucros de empresas com maior grau de intangibilidade. Como já abordado na seção anterior, para Scott (2012), os ativos intangíveis são gerados internamente e seus custos são diluídos ao longo dos anos, tornando mais complexa sua adequada mensuração e reconhecimento. Além disso, destacase a subjetividade atrelada à segregação entre gastos operacionais e investimentos em intangíveis e a incerteza atrelada à mensuração dos benefícios econômicos futuros (Kanodia et al., 2004). Diante disso, a complexidade e incerteza dos intangíveis podem afetar negativamente a previsibilidade dos lucros futuros. Conforme Barron, Byard, Kile e Riedl (2002), Gu e Wang (2005) e Dinh et al. (2015), a dificuldade em avaliá-los tende a reduzir a qualidade das previsões.

Diante do exposto, acredita-se que a complexidade dos intangíveis está associado à acurácia das previsões dos analistas, aumentando ou reduzindo a precisão das previsões de lucros realizada pelos analistas financeiros em função do nível da complexidade de cada intangível estudado, gerando a primeira hipótese de pesquisa.

H1 - A acurácia das previsões dos analistas financeiros será maior quando associada aos intangíveis com menor grau de incerteza e complexidade.

\subsection{Dispersão das previsões dos analistas e os ativos intangíveis}

Em consonância com outros estudos (Dinh et al., 2015; Jia, 2017; Kwon, 2002; Matolcsy \& Wyatt, 2006; Srinivasan, 2007), também será investigada a influência dos intangíveis sobre a dispersão das previsões dos analistas. Kwon (2002) e Matolcsy e Wyatt (2006) encontraram menor grau de dispersão em empresas com mais intangíveis, enquanto Dinh et al. (2015) e Jia (2017) utilizaram a dispersão em seus trabalhos e concluíram que o investimento em intangíveis está significativamente associado a maiores níveis de dispersão das previsões. Srinivasan (2007) explica que tal métrica é de grande interesse para usuários internos e externos de empresas participantes do mercado de ações por refletir a variabilidade ex-ante do desempenho da empresa.

No que diz respeito à sua relação com os intangíveis, a dispersão da previsão é capaz de refletir a incerteza gerada pelos intangíveis sobre os lucros futuros da empresa, a qualidade da informação, podendo também ser interpretada como uma medida alternativa de assimetria de informação no mercado de capitais (Barron et al., 1998; Chakravarty \& Grewal, 2016). Seguindo a mesma perspectiva abordada para a construção da primeira hipótese, empresas com maior grau de intangibilidade atraem uma maior cobertura e, dessa forma, aumentam a acessibilidade de informações (Kwon, 2002). Para Barth et al. (2001), empresas com maior nível de intangível fornecem maiores incentivos aos analistas por possibilitarem maiores chances de ganhos. Ou seja, empresas com mais intangíveis geram uma maior disponibilidade de informações a todos usuários, podendo reduzir a assimetria informacional, tornando as previsões menos dispersas.

Como exemplo, Matolcsy e Wyatt (2006), ao verificarem empresas australianas no período de 1990 a 1997, encontraram evidências de que a capitalização dos intangíveis está associada à maior acurácia das previsões e menor grau de dispersão. Como outro exemplo, pode-se citar Kwon (2002), que segregou sua amostra em dois grupos: (i) high-tech e (ii) low-tech e, com isso, examinou as diferenças de acurácia e dispersão entre os grupos. Os resultados apontaram maior acurácia e menor dispersão para empresas pertencentes ao grupo high-tech em relação às empresas low-tech.

Em contraste com esses trabalhos, outros autores argumentam que as informações relacionadas aos intangíveis são consideradas como benefícios econômicos futuros incertos que também podem refletir na dispersão das previsões (Amir, Lev \& Sougiannis, 2007; Dinh et al., 2015; Gu \& Wang, 2005). Chakravarty e Grewal (2016) explicam que, à medida que a qualidade da informação reduz, torna-se mais difícil a previsão das perspectivas de crescimento e, então, os modelos de avaliação não conseguem prever adequadamente os lucros futuros. 
Segundo Dinh et al. (2015), existem dois níveis de incerteza relacionadas aos intangíveis. Primeiramente, a incerteza do ambiente em que a empresa opera, onde não é possível prever as ações de clientes, fornecedores e concorrentes, que podem afetar a produção, vendas e o desempenho. Em segundo lugar, deve-se citar a incerteza relacionada aos benefícios econômicos futuros, uma vez que o sucesso de um intangível dificilmente pode ser previsto (Amir, Lev \& Sougiannis, 2007; Dinh et al., 2015; Gu \& Wang, 2005). Dinh et al. (2015), com dados das 150 maiores empresas da bolsa de valores alemã, mostram que a capitalização dos custos de pesquisa e desenvolvimento (P\&D) está significativamente associada aos erros de previsão dos analistas mais altos e à dispersão de tal previsão.

Com base nestes argumentos, acredita-se que a incerteza dos intangíveis está associada às dispersões das previsões dos analistas, aumentando ou reduzindo a dispersão dos prognósticos de lucros realizadas pelos analistas financeiros em função do nível de incerteza de cada intangível estudado, o que culmina na segunda hipótese de pesquisa.

H2 - A dispersão das previsões dos analistas financeiros será menor quando associada aos intangíveis com menor grau de incerteza e complexidade.

\section{METODOLOGIA}

\subsection{Coleta de dados e amostra}

A amostra da pesquisa é composta por todas as empresas norte-americanas não financeiras com ações listadas na NASDAQ no período de 1995 a 2016 e disponibilizadas pela base de dados Thomson Reuters. A escolha do período da amostra deu-se pelo período com maior número de analistas fornecendo previsões. A escolha pela respectiva bolsa de valores deu-se, primeiramente, em virtude da maior disponibilidade de dados dos intangíveis estudados e pela maior cobertura de analistas, pela comparabilidade com outros resultados existentes na literatura e pelo fato de que, conforme Burgman e Roos (2004), a NASDAQ concentra as empresas listadas da nova economia com um maior grau de intangibilidade, foco deste trabalho. A relação número de empresas/ano após a delimitação da amostra é apresentada na Tabela 1, a seguir:

Tabela 1

Número de empresas/ano

\begin{tabular}{cccccccccccc}
\hline Ano & Empresas & $\%$ & Ano & Empresas & $\%$ & Ano & Empresas & \% & Ano & Empresas & $\%$ \\
\hline 1995 & 1583 & $2.80 \%$ & 2001 & 2615 & $4.63 \%$ & 2007 & 3461 & $6.13 \%$ & 2013 & 3929 & $6.96 \%$ \\
1996 & 2125 & $3.76 \%$ & 2002 & 2664 & $4.72 \%$ & 2008 & 3560 & $6.31 \%$ & 2014 & 3902 & $6.91 \%$ \\
1997 & 2314 & $4.10 \%$ & 2003 & 2733 & $4.84 \%$ & 2009 & 3634 & $6.44 \%$ & 2015 & 3723 & $6.59 \%$ \\
1998 & 2447 & $4.33 \%$ & 2004 & 2366 & $4.19 \%$ & 2010 & 3723 & $6.59 \%$ & 2016 & 3531 & $6.25 \%$ \\
1999 & 2547 & $4.51 \%$ & 2005 & 3019 & $5.35 \%$ & 2011 & 3851 & $6.82 \%$ & & & \\
2000 & 2598 & $4.60 \%$ & 2006 & 3214 & $5.69 \%$ & 2012 & 3938 & $6.97 \%$ & Total & $\mathbf{5 6 , 4 6 1}$ & $\mathbf{1 0 0 \%}$ \\
\hline
\end{tabular}

Fonte: Elaborado pelos autores.

Para delimitação e exclusão de empresas muito pequenas da base, apresentadas na Tabela 1, foram excluídas as com menos de US\$ 1 milhão em vendas e em ativos totais (Barth, Kasznik, \& McNichols, 2001; Palmon \& Yezegel, 2012). Observa-se que houve um aumento no número de empresas encaixadas em tais delimitações até o ano de 2012: saindo de 1583 empresas em 1995 e chegando em um total de 3938 empresas em 2012. Posteriormente, houve uma redução do número de empresas ao longo dos anos, chegando a 3531 em 2016. 


\subsection{Variáveis utilizadas}

A partir da observação dos estudos que relacionam as previsões dos analistas aos intangíveis, identificaram-se as variáveis dependentes, independentes e de controle, assim como seus métodos de mensuração. Em virtude da grande variedade de métodos de aferição das variáveis, ressalta-se que, para escolha das variáveis e forma de mensuração utilizada, observouse as proxies mais utilizadas para a mensuração das previsões dos analistas financeiros e do ativo intangível. Na Tabela 2 é apresentado um resumo das variáveis.

Tabela 2

\section{Definição das variáveis}

\begin{tabular}{lcll}
\hline \multicolumn{1}{c}{ Variável } & Abreviação & \multicolumn{1}{c}{ Definição/Método de cálculo } & \multicolumn{1}{c}{ Literatura } \\
\hline $\begin{array}{l}\text { Variáveis dependentes } \\
\text { Acurácia das previsões }\end{array}$ & APN & $\begin{array}{l}\text { Negativo do valor absoluto dos erros de } \\
\text { previsão dos analistas deflacionados pelo } \\
\text { preço das ações. }\end{array}$ & $\begin{array}{l}\text { Almeida e Dalmácio, 2015; } \\
\text { Dalmácio, 2009; Gu e Wang, } \\
\text { 2005; Jia, 2017; Lang e } \\
\text { Lundholm, 1996. }\end{array}$ \\
$\begin{array}{lll}\text { Dispersão das } \\
\text { previsões dos analistas }\end{array}$ & DP & $\begin{array}{l}\text { Desvio padrão das estimativas de } \\
\text { previsão para empresa i no período t, } \\
\text { escalonado pelo preço da ação da } \\
\text { empresa i no período t. }\end{array}$ & $\begin{array}{l}\text { Almeida e Dalmácio, 2015; } \\
\text { Dinh et al., 2015; Jia, 2017; } \\
\text { Lang e Lundholm, 1996; } \\
\text { Srinivasan, 2007. }\end{array}$ \\
\hline
\end{tabular}

Variáveis independentes

$\begin{array}{ll}\text { Goodwill } & \text { GW } \quad \begin{array}{l}\text { Goodwill líquido dividido pelo ativo total } \\ \text { da empresa i em } t .\end{array}\end{array}$

P\&D $\quad$ IPD

Investimento total em P\&D dividido pelo Shen, 2013; Palmon e total de vendas da empresa i no período t. Yezegel, 2012; Srinivasan, 2007.

\begin{tabular}{|c|c|c|c|}
\hline Ativo intangível & AI & $\begin{array}{l}\text { Mercado da empresa i no período t } \\
\text { (MVAD) }\end{array}$ & Matolcsy e Wyatt, 2006 \\
\hline \multicolumn{4}{|l|}{ Variáveis de controle } \\
\hline Tamanho & TAM & $\begin{array}{l}\text { Logaritmo natural do valor de mercado } \\
\text { da empresa i no período t. }\end{array}$ & $\begin{array}{l}\text { Amir } \text { et al., 2003; Dinh et al., } \\
\text { 2015; Gu e Wang, 2005; } \\
\text { Matolcsy e Wyatt, } 2006 .\end{array}$ \\
\hline Setor & D_SET & $\begin{array}{l}\text { Setor de atividade da empresa }(1= \\
\text { empresas de determinado setor e } 0=\text { para } \\
\text { empresas de outros setores })\end{array}$ & Jia, 2017 \\
\hline $\begin{array}{l}\text { Dummy lucro líquido } \\
\text { negativo }\end{array}$ & D_LLN & $\begin{array}{l}1=\text { empresas com lucro líquido negativo } \\
\text { e } 0=\text { outras empresas }\end{array}$ & $\begin{array}{l}\text { Amir et al., 2003; Gu e } \\
\text { Wang, } 2005\end{array}$ \\
\hline Cobertura dos analistas & CA & $\begin{array}{l}\text { Número de analistas que emitiram } \\
\text { previsões de ganho à empresa i no } \\
\text { período t. }\end{array}$ & $\begin{array}{l}\text { Gu e Wang, 2005; Dinh et al., } \\
\text { 2015; Jia, 2017; Palmon e } \\
\text { Yezegel, 2012; Srinivasan, } \\
\text { 2007. }\end{array}$ \\
\hline Dummy de ano & ANO & $\begin{array}{l}\text { Dummy ano da amostra que assume } \\
\text { valor igual a um para determinado ano e } \\
\text { zero para outros anos }\end{array}$ & Jia, 2017 \\
\hline $\begin{array}{l}\text { Crescimento das } \\
\text { vendas }\end{array}$ & GROW & Crescimento da receita & Barth et al., 2001; Jia, 2017. \\
\hline Rentabilidade do ativo & ROA & Rentabilidade sobre ativo total & Jia, 2017 \\
\hline
\end{tabular}
Fonte: Original desta pesquisa.

Destacando-se a mensuração das variáveis das previsões dos analistas, a acurácia da previsão (AP) é medida como o valor negativo da diferença absoluta entre a mediana da previsão dos analistas (diferença absoluta entre a previsão consensual dos analistas e o lucro por ação real anual da empresa no período), deflacionado pelo preço das ações no último dia útil. (Almeida \& Dalmácio, 2015; Lang \& Lundholm, 1996). Segundo Matolcsy e Wyatt (2006), a variável é 
desinflada para facilitar comparações entre empresas. Já a dispersão das previsões (DP) é calculada como o desvio padrão das estimativas de previsão para empresa i no período $t$, escalonado pelo preço da ação da empresa i no período t (Almeida \& Dalmácio, 2015; Jia, 2017; Kwon, 2002). Em ambas variáveis, a literatura também aponta outras opções de divisor, como Matolcsy e Wyatt (2006), os quais utilizam o ativo total, e Kwon (2002), que utiliza a média das previsões dos analistas. Entretanto, observa-se a maior utilização do preço das ações no fim do exercício como divisor (Almeida \& Dalmácio, 2015; Dinh et al. 2015; Jia, 2017; Lang \& Lundholm, 1996; Srinivasan, 2007).

\subsection{Modelos}

Para facilitar o entendimento, foram elaborados dois modelos gerais para a investigação da relação entre os ativos intangíveis e as previsões dos analistas. No modelo 1, utilizou-se como variável dependente a "acurácia das previsões dos analistas" e as variáveis independentes que representam os intangíveis, apresentadas na Tabela 2 (P\&D, Ativos intangíveis e Goodwill), em regressões distintas. Enquanto no modelo 2, por outro lado, utilizou-se a "dispersão das previsões dos analistas" como variável dependente do modelo e, novamente, como variáveis independentes foram utilizados o "P\&D", "Ativos intangíveis" e "Goodwill".

$$
A P_{i t}=\beta_{0}+\beta_{1} \text { Intangível }_{i t}+\beta_{2} \text { Control }_{i t}+e_{i t}
$$

Equação (1)

Em que, $A P_{i t}$ : acurácia das previsões dos analistas pelo consenso (média e mediana) dos analistas da empresa i em t; Intangível $_{i t}=$ variável de intangível da empresa i em t; Control $_{i t}=$ variáveis de controle da empresa i em t; $e_{i t}=$ erro aleatório para a empresa $\mathrm{i}$, em t.

$$
D P_{i t}=\beta_{0}+\beta_{1} \text { Intangível }_{i t}+\beta_{2} \text { Control }_{i t}+e_{i t}
$$

Equação (2)

Em que, $D P_{i t}=$ dispersão das previsões dos analistas da empresa i em t; Intangível $_{i t}=$ variável de intangível da empresa i em t; Control $_{i t}=$ variáveis de controle da empresa i em t; $e_{i t}=$ erro aleatório para a empresa i em t.

Isto é, na primeira regressão econométrica, foi investigado o impacto ativo intangível como variável independente sobre a acurácia das previsões dos analistas. Na segunda regressão, foi investigado o impacto do $\mathrm{P} \& \mathrm{D}$ como variável independente sobre a acurácia das previsões dos analistas. E, por último, foi investigado o impacto do "Goodwill" como variável independente sobre a acurácia das previsões dos analistas. Sendo assim, tais modelos originam a seis modelos distintos a partir da alternância das variáveis estudadas.

\section{ANÁLISE DOS RESULTADOS}

\subsection{Análise das Estatísticas Descritivas e Correlações}

Nesta seção, será apresentada a estatística descritiva das variáveis da amostra desta pesquisa. Ressalta-se que, em virtude da alta dispersão das variáveis, foi utilizado o processo de winsorização, que é um processo para limitar os efeitos de outliers, sem removê-los (Fusai \& Roncoroni, 2007). Na Tabela 3, é apresentada a estatística descritiva das variáveis já winsorizadas, tendo em vista que, ao observar as variáveis independentes de intangível "Goodwill", "P\&D" e "ativo intangível", verifica-se a existência de empresas com pouco investimento em P\&D. 
Tabela 3

\section{Estatística Descritiva}

\begin{tabular}{lccccc}
\multicolumn{1}{c}{ Variáveis } & Número de observações & Média & Desvio Padrão & Mínimo & Máximo \\
\hline AP & 34,167 & -1.936 & 24.79 & -399.2 & 0 \\
DP & 34,314 & 0.131 & 1.929 & 0 & 33.33 \\
GW & 35,638 & 0.192 & 0.248 & 0 & 1.731 \\
AI & 36,841 & 0.117 & 0.576 & -2.634 & 3.063 \\
IPD & 25,142 & 0.533 & 2.655 & 0 & 117.1 \\
TAM & 60,183 & 19.20 & 3.318 & 6.076 & 23.93 \\
GROW & 64,652 & 0.398 & 1.776 & -1 & 17.01 \\
D_PREJ & 67,977 & 0.342 & 0.474 & 0 & 1 \\
ROA & 67,910 & -0.0790 & 0.593 & -36.68 & 0.495 \\
CA & 38,144 & 1.707 & 0.984 & 0 & 4.025 \\
\hline NAtaP
\end{tabular}

Notas. AP = acurácia das previsões dos analistas medida pela média dos analistas da empresa i em t; AP_MEDIAN = acurácia das previsões dos analistas medida pela mediana dos analistas da empresa i em t; DP = desvio padrão das estimativas de previsão para empresa i no período $t$, escalonado pelo preço da ação da empresa i no período t; GW = valor de Goodwill líquido dividido pelo ativo total da empresa i em t; IPD = investimento total em P\&D dividido pelo total de vendas da empresa i no período t; $\mathrm{AI}=$ ativos intangíveis reconhecidos mensurados pelo ativo Intangível dividido por MVAD (valor de mercado adicionado da empresa i em t); TAM = logaritmo natural do valor de mercado da empresa; D_PREJ = dummy para empresas com prejuízo onde $1=$ empresas com lucro líquido negativo e $0=$ outras empresas; $\mathrm{CA}=$ logaritmo natural do número de analistas que emitiram previsões de ganho à empresa i no período t; GROW = crescimento da receita da empresa $\mathrm{i}$ em $\mathrm{t}$ em relação à $\mathrm{t}-1 ; \mathrm{ROA}=$ rentabilidade sobre ativo total da empresa i em t; Ctrl. setor $=$ Setor de atividade da empresa $(1=$ empresas de determinado setor e $0=$ para empresas de outros setores); Ctrl. ano = dummy ano da amostra que assume valor igual a um para determinado ano e zero para outros anos.

Pelos dados apresentados na Tabela 3, nota-se a ocorrência de empresas com pouquíssimo investimento em P\&D (IPD). Destaca-se, também, o fato de que as variáveis dependentes (AP e DP) possuem interpretações distintas. A acurácia das previsões apresenta seu máximo zero, demonstrando que, quanto maior e mais próximo de zero, mais precisa é a previsão. Já a dispersão apresenta seu valor mínimo zero, indicando que, quanto menor e mais próximo de zero, menor será a dispersão entre as previsões. Um cuidado adicional que deve ser tomado, além da observação das estatísticas descritivas, antes da realização dos testes, constitui-se pela análise de correlação. Segundo Dalmácio (2009), a partir desta verificação, é possível realizar uma análise preliminar das relações existentes entre as variáveis. Dessa forma, na Tabela 4 é apresentada a matriz de correlação entre as variáveis para observar o grau de associação entre as variáveis.

Tabela 4

\section{Matriz de correlação das variáveis}

\begin{tabular}{|c|c|c|c|c|c|c|c|c|c|c|}
\hline & $\mathrm{AP}$ & DP & IPD & $\mathrm{AI}$ & TAM & GROW & D_PREJ & ROA & $\mathrm{CA}$ & GW \\
\hline AP & 1 & & & & & & & & & \\
\hline DP & $-.655 * * *$ & 1 & & & & & & & & \\
\hline IPD & -.005 & .004 & 1 & & & & & & & \\
\hline AI & $.060 * * *$ & $-.035 * *$ & .002 & 1 & & & & & & \\
\hline TAM & $.263 * * *$ & $-.238 * * *$ & $-.048 * * *$ & -.0176 & 1 & & & & & \\
\hline GROW & -.020 & $.024 *$ & $.071 * * *$ & -.0012 & $-.075 * * *$ & 1 & & & & \\
\hline D_PREJ & $-.074 * * *$ & $.072 * * *$ & $.138 * * *$ & $.038 * * *$ & $-.429 * * *$ & $.064 * * *$ & 1 & & & \\
\hline ROA & $.239 * * *$ & $-.121 * * *$ & $-.165 * * *$ & -.021 & $.365 * * *$ & $-.100 * * *$ & $-.511 * * *$ & 1 & & \\
\hline $\mathrm{CA}$ & $.058 * * *$ & $-.023 *$ & -.007 & $-.0413 * * *$ & $.571 * * *$ & $-.0535 * * *$ & $-.207 * * *$ & $.189 * * *$ & 1 & \\
\hline GW & .009 & -.007 & $-.034 * *$ & $.104 * * *$ & $.066 * * *$ & $.086 * * *$ & .006 & $.027 *$ & $.127 * * *$ & 1 \\
\hline
\end{tabular}

Notas. Os asteriscos indicam a significância: *** $(1 \%), * *(5 \%)$ e * $(10 \%)$. AP = acurácia das previsões dos analistas medida pela mediana dos analistas da empresa i em $\mathrm{t}$; $\mathrm{DP}=$ desvio padrão das estimativas de previsão para empresa $\mathrm{i}$ 
no período t, escalonado pelo preço da ação da empresa i no período t; IPD = investimento total em P\&D dividido pelo total de vendas da empresa i no período t; $\mathrm{AI}=$ ativos intangíveis reconhecidos mensurados pelo ativo Intangível dividido por MVAD (valor de mercado adicionado da empresa i em t); TAM = logaritmo natural do valor de mercado da empresa; D_PREJ = dummy para empresas com prejuízo onde $1=$ empresas com lucro líquido negativo e $0=$ outras empresas; $\mathrm{CA}=$ logaritmo natural do número de analistas que emitiram previsões de ganho à empresa i no período t; GROW = crescimento da receita da empresa $\mathrm{i}$ em $\mathrm{t}$ em relação à $\mathrm{t}-1 ; \mathrm{ROA}=$ rentabilidade sobre ativo total da empresa i em t; Ctrl. setor $=$ Setor de atividade da empresa $(1=$ empresas de determinado setor e $0=$ para empresas de outros setores); Ctrl. ano = dummy ano da amostra que assume valor igual a 1 (um) para determinado ano e 0 (zero) para outros anos e GW = valor de Goodwill líquido dividido pelo ativo total da empresa i em t.

Por meio da matriz de correlação, assim, foi possível observar que as variáveis dependentes AP e DP, como esperado, apresentam correlação negativa com significância estatística, o que pode indicar que as previsões de lucros melhores acarretam maior precisão e menor dispersão entre as previsões dos analistas. A correlação das variáveis independentes e as de controle, com as variáveis dependentes, apresentam correlação com significância estatística, exceto entre IDP, GROW e GWNET e a variável dependente AP_MEDIAN. Todavia, com relação à variável dependente DP, somente as variáveis IDP e GWNET não apresentam significância estatística na correlação. Também se observa que as variáveis independentes não possuem alta correlação entre si, o que poderia ser um indício de problemas de endogeneidade.

\subsection{Análise dos testes}

Para avaliar a relação dos ativos intangíveis e as previsões dos analistas financeiros, foram aplicados os modelos citados, de modo que os prognósticos são representados pelas variáveis dependentes de acurácia das previsões (AP) e dispersão das previsões (DP). As proxies para os ativos intangíveis são representadas pelos investimentos em P\&D (IPD), ativos intangíveis reconhecidos nas demonstrações financeiras (AI) e o Goodwill (GW). Na Tabela 5, encontram-se os resultados das análises econométricas com dados em painel utilizando-se o método OLS (Ordinary Least Squares - Mínimos Quadrados Ordinários), conforme adotado pela maior parte da literatura internacional encontrada acerca do tema (Amir et al., 2003; Jia, 2017; Matolcsy; \& Wyatt, 2006). Nas regressões realizadas, foi utilizado o erro-padrão robusto, pois, conforme Fávero e Belfiore (2014, p. 159), possibilita-se, desse modo, a obtenção de estimadores não enviesados.

Tabela 5

\section{Resultados}

\begin{tabular}{|c|c|c|c|c|c|c|}
\hline $\begin{array}{l}\text { Modelo } \\
\text { Variáveis }\end{array}$ & $\begin{array}{l}\text { (1) } \\
\text { AP }\end{array}$ & $\begin{array}{l}\text { (2) } \\
\text { DP }\end{array}$ & $\begin{array}{l}\text { (3) } \\
\text { AP }\end{array}$ & $\begin{array}{l}\text { (4) } \\
\text { DP }\end{array}$ & $\begin{array}{l}\text { (5) } \\
\text { AP }\end{array}$ & $\begin{array}{l}\text { (6) } \\
\text { DP }\end{array}$ \\
\hline IPD & $\begin{array}{c}0.119 * * * \\
(2.787)\end{array}$ & $\begin{array}{c}-0.00352 \\
(-0.677)\end{array}$ & & & & \\
\hline AI & & & $\begin{array}{c}0.750 * \\
(1.658)\end{array}$ & $\begin{array}{c}-0.0574 * \\
(-1.773)\end{array}$ & & \\
\hline GW & & & & & $\begin{array}{c}-3.481 * * * \\
(-2.935)\end{array}$ & $\begin{array}{c}0.158 * * \\
(2.336)\end{array}$ \\
\hline TAM & $\begin{array}{c}4.003 * * * \\
(8.204)\end{array}$ & $\begin{array}{c}-0.375 * * * \\
(-7.846)\end{array}$ & $\begin{array}{c}3.337 * * * \\
(8.975)\end{array}$ & $\begin{array}{c}-0.235 * * * \\
(-7.610)\end{array}$ & $\begin{array}{c}4.035 * * * \\
(9.321)\end{array}$ & $\begin{array}{c}-0.258 * * * * \\
(-7.402)\end{array}$ \\
\hline D_PREJ & $\begin{array}{c}4.199 * * * \\
(5.689)\end{array}$ & $\begin{array}{c}-0.345 * * * \\
(-5.919)\end{array}$ & $\begin{array}{c}3.057 * * * \\
(4.532)\end{array}$ & $\begin{array}{c}-0.226 * * * \\
(-3.207)\end{array}$ & $\begin{array}{c}3.667 * * * \\
(4.946)\end{array}$ & $\begin{array}{c}-0.184 * * * \\
(-3.942)\end{array}$ \\
\hline GROW & $\begin{array}{c}0.200 \\
(1.175)\end{array}$ & $\begin{array}{c}0.00325 \\
(0.211)\end{array}$ & $\begin{array}{c}-0.123 \\
(-0.450)\end{array}$ & $\begin{array}{c}-0.00425 \\
(-0.223)\end{array}$ & $\begin{array}{l}-0.0522 \\
(-0.161)\end{array}$ & $\begin{array}{l}0.0105 \\
(0.452)\end{array}$ \\
\hline $\mathrm{ROA}$ & $\begin{array}{c}2.974 \\
(1.538)\end{array}$ & $\begin{array}{l}-0.0178 \\
(-0.161)\end{array}$ & $\begin{array}{c}6.131 * * \\
(2.192)\end{array}$ & $\begin{array}{c}-0.623 * * \\
(-2.029)\end{array}$ & $\begin{array}{c}6.576 * * \\
(1.993)\end{array}$ & $\begin{array}{c}-0.276 \\
(-1.382)\end{array}$ \\
\hline $\mathrm{CA}$ & $\begin{array}{c}-4.116 * * * \\
(-7.518)\end{array}$ & $\begin{array}{c}0.482 * * * \\
(7.722)\end{array}$ & $\begin{array}{c}-2.878 * * * \\
(-8.221)\end{array}$ & $\begin{array}{c}0.263 * * * \\
(7.680)\end{array}$ & $\begin{array}{c}-3.379 * * * \\
(-8.501)\end{array}$ & $\begin{array}{c}0.276^{* * * *} \\
(7.288)\end{array}$ \\
\hline Constante & $\begin{array}{c}-144.7 * * * \\
(-4.137)\end{array}$ & $\begin{array}{c}10.66^{* * *} * \\
(3.845)\end{array}$ & $\begin{array}{c}-122.1 * * * \\
(-4.474)\end{array}$ & $\begin{array}{c}7.530 * * * \\
(3.451)\end{array}$ & $\begin{array}{c}-93.00 * * * \\
(-4.231)\end{array}$ & $\begin{array}{c}6.109 * * * \\
(2.879)\end{array}$ \\
\hline Crtl. Ano & Sim & Sim & Sim & Sim & Sim & Sim \\
\hline
\end{tabular}




\begin{tabular}{lcccccc} 
Crtl Setor & Sim & Sim & Sim & Sim & Sim & Sim \\
Observações & 13,378 & 13,418 & 23,704 & 23,768 & 23,375 & 23,436 \\
R2 Ajust. & 0.142 & 0.132 & 0.126 & 0.0965 & 0.134 & 0.0916 \\
F & 1.739 & 1.456 & 2.014 & 1.436 & 2.143 & 1.355 \\
\hline
\end{tabular}

Notas. Os asteriscos indicam a significância: *** (1\%), ** (5\%) e * (10\%). Os valores entre parênteses é o t-statistic dos coeficientes estimados. AP = acurácia das previsões dos analistas medida pela mediana dos analistas da empresa i em t; DP = desvio padrão das estimativas de previsão para empresa i no período t, escalonado pelo preço da ação da empresa i no período t; IPD = investimento total em P\&D dividido pelo total de vendas da empresa i no período t; AI = ativos intangíveis reconhecidos mensurados pelo ativo Intangível dividido por MVAD (valor de mercado adicionado da empresa i em t); GW = valor de Goodwill líquido dividido pelo ativo total da empresa i em t; TAM = logaritmo natural do valor de mercado da empresa; D_PREJ = dummy para empresas com prejuízo onde $1=$ empresas com lucro líquido negativo e $0=$ outras empresas; $\overline{C A}=$ logaritmo natural do número de analistas que emitiram previsões de ganho à empresa $\mathrm{i}$ no período $\mathrm{t}$; GROW = crescimento da receita da empresa $\mathrm{i}$ em $\mathrm{t}$ em relação à t-1; ROA = rentabilidade sobre ativo total da empresa i em t; Ctrl. setor $=$ Setor de atividade da empresa $(1=$ empresas de determinado setor e $0=$ para empresas de outros setores); Ctrl. ano = dummy ano da amostra que assume valor igual a um para determinado ano e zero para outros anos.

Nas regressões 1 e 2, que tiveram o objetivo de analisar especificamente a influência do intangível de investimento em P\&D sobre as previsões dos analistas, representadas pelas variáveis AP e DP, verificou-se que a variável IPD apresentou impacto positivo $(0,119)$ e significância estatística $(1 \%)$ sobre a variável $\mathrm{AP}$, mas não apresentou significância estatística em relação à dispersão das previsões dos analistas (DP). Com isso, as evidências sugerem que os investimentos em P\&D exercem influência positiva sobre a acurácia das previsões.

As regressões 3 e 4 foram realizadas visando investigar a influência dos ativos intangíveis reconhecidos nas demonstrações financeiras (AI) sobre as previsões dos analistas (AP e DP). Os resultados apontaram impacto positivo $(0,750)$ e significância estatística $(10 \%)$ sobre a variável $\mathrm{AP}$, sugerindo que os ativos intangíveis reconhecidos nas demonstrações financeiras também exercem influência positiva sobre a acurácia das previsões. Por sua vez, a variável AI apresentou valor negativo $(-0,0566)$ e estatisticamente significante $(10 \%)$ sobre a dispersão das previsões dos analistas (DP), sugerindo que um aumento nos intangíveis reconhecidos nas demonstrações (AI) reduz a dispersão das precisões dos analistas (DP). Portanto, os achados sugerem que os ativos intangíveis reconhecidos nas demonstrações influenciam positivamente as previsões dos analistas, reduzindo a dispersão dos prognósticos.

Nota-se que os resultados referentes aos intangíveis de investimentos em $P \& D$ e ativos intangíveis reconhecidos nas demonstrações apontam para uma melhora significativa das previsões dos analistas, apesar dos investimentos em P\&D serem considerados intangíveis "mais incertos" devido à dificuldade de prever ou controlar seu sucesso (Amir et al., 2003; Dinh et al., 2015; Gu \& Wang, 2005). Conforme Diamond e Verrechia (1991), Lang e Lundholm (1996) e Srinivasan (2007), o maior volume de informações a respeito do fluxo de caixa futuro, gerado pelo reconhecimento dos investimentos em $\mathrm{P} \& \mathrm{D}$, tende a melhorar as previsões dos analistas.

Quanto aos resultados referentes à dispersão das previsões dos analistas (DP), apesar de os investimentos em $\mathrm{P} \& \mathrm{D}$ não apresentarem significância estatística, os ativos intangíveis reconhecidos nas demonstrações (AI) apresentaram associação com a redução da incerteza gerada pelos intangíveis, uma vez que a dispersão, segundo Barron et al. (1998), é capaz de refletir tal incerteza. Tal resultado vai de encontro com o apontado pela teoria quando indica que apenas aqueles intangíveis que se aproximem mais dos tangíveis são reconhecidos pela contabilidade como ativos intangíveis (Zanoteli, 2015).

Objetivando contribuir não somente com as variáveis identificadas na literatura existente, que relaciona o intangível à previsão dos analistas, buscou-se adicionar outra variável ao estudo: o Goodwill (GW). No resultado das regressões econométricas 5 e 6, apresentadas na Tabela 5, verificou-se que o GW se mostrou estatisticamente significante no modelo relacionado com a acurácia das previsões dos analistas (AP) e no modelo da dispersão das previsões (DP), respectivamente a $1 \%$ e $5 \%$. Entretanto, diferentemente das outras variáveis estudadas, o GW 
apresentou valor negativo (-3.481) quando relacionado com AP e valor positivo $(0,158)$ quando relacionado à dispersão das previsões (DP).

Dessa forma, as evidências sugerem que o Goodwill tende a influenciar negativamente as previsões dos analistas, diminuindo a acurácia e aumentando a dispersão. Isso sugere que o Goodwill é uma informação com maior complexidade e incerteza, podendo afetar negativamente a previsibilidade dos lucros futuros, o que corrobora com as pesquisas de Barron et al. (2002), Gu e Wang (2005) e Dinh et al. (2015), as quais sugerem a dificuldade de avaliar os intangíveis como um fator preponderante para a redução da qualidade das previsões dos analistas.

\subsection{Resultados esperados vs. Resultados encontrados}

É importante ressaltar que o sinal do coeficiente esperado pode diferir de acordo com a incerteza, complexidade e quantidade de informações do ativo intangível estudado. Zanoteli (2015) ressalta que as premissas para o reconhecimento contábil estão alinhadas ao conceito de bens tangíveis, sugerindo um desalinhamento com a definição de intangível. Ou seja, espera-se que apenas aqueles intangíveis que se aproximem mais dos tangíveis (menos incertos) sejam reconhecidos pela Contabilidade. Dessa forma, consideram-se os ativos intangíveis reconhecimentos como intangíveis com um menor grau de incerteza. Por outro lado, as atividades de P\&D são mais incertas e complexas em relação aos ativos intangíveis reconhecidos, uma vez que o sucesso de uma atividade de P\&D é difícil de controlar e prever (Amir et al., 2003; Dinh et al., 2015; Gu \& Wang, 2005). Dinh et al. (2015) explicam, portanto, que os investimentos em $\mathrm{P} \& \mathrm{D}$ estão associados ao aumento da assimetria informacional devido à incerteza dos benefícios econômicos futuros e à volatilidade dos lucros subsequentes.

Esperou-se que os investimentos em $\mathrm{P} \& \mathrm{D}$ estivessem associados à redução da previsibilidade dos lucros (redução da acurácia e aumento da dispersão), enquanto os ativos intangíveis reconhecidos com o aumento da previsibilidade e consequente melhoria das previsões (aumento da acurácia e redução da dispersão), em virtude da já apresentada maior incerteza inerente aos investimentos em P\&D. Entretanto, a não confirmação dos sinais esperados não inviabiliza a confirmação ou rejeição das hipóteses de pesquisa. Conforme já apresentado, a maior divulgação de informações relacionadas aos benefícios futuros gerados pelos ativos intangíveis pode ser capaz de melhorar as previsões dos analistas (Diamond \& Verrecchia, 1991; Lang \& Lundholm, 1996; Srinivasan, 2007). Ou seja, uma maior divulgação dos investimentos em P\&D pode resultar na compensação da incerteza por meio do volume informacional. A seguir, a Tabela 6 apresenta um resumo dos resultados esperados e encontrados nos testes realizados no trabalho:

Tabela 6

Resultados esperados vs. Encontrados

\begin{tabular}{ccccccc}
\hline Hipótese & Modelo & $\begin{array}{c}\text { Variável } \\
\text { Dependente }\end{array}$ & $\begin{array}{c}\text { Variável } \\
\text { Independente }\end{array}$ & $\begin{array}{c}\text { Coeficiente } \\
\text { esperado }\end{array}$ & Abordagem & $\begin{array}{c}\text { Coeficiente } \\
\text { encontrado }\end{array}$ \\
\hline H1 & 1 & Acurácia (AP) & IPD & $(-)$ & OLS & $(+)$ \\
H2 & 2 & Dispersão (DP) & IPD & $(+)$ & OLS & Não significante \\
H1 & 3 & Acurácia (AP) & AI & $(+)$ & OLS & $(+)$ \\
H2 & 4 & Dispersão (DP) & AI & $(-)$ & OLS & $(-)$ \\
H1 & 5 & Acurácia (AP) & GW & $(-)$ & OLS & $(-)$ \\
H2 & 6 & Dispersão (DP) & GW & $(+)$ & OLS & $(+)$ \\
\hline
\end{tabular}

Fonte: Elaborado pelos autores.

Quando comparados os coeficientes esperados com os encontrados, observa-se que o investimento em P\&D (IPD) mostrou-se significante estatisticamente no modelo 1. Entretanto, de forma inesperada, a variável apresentou-se positivamente relacionada com a acurácia das previsões. Já o ativo intangível (AI) reconhecido, por sua vez, mostrou-se significante em ambos os modelos analisados (modelos 3 e 4) e, como esperado, a variável apresentou-se positivamente 
relacionada com a acurácia das previsões dos analistas financeiros (AP) e demonstrou associação negativa com a dispersão das previsões dos analistas (DP). Dessa forma, os resultados entre os intangíveis e a acurácia das previsões de lucros dos analistas evidenciam uma melhora significativa das previsões, tanto pela variável IPD quanto pela variável AI, indicando que os investimentos em P\&D também podem contribuir para a previsibilidade dos lucros futuros. Com relação aos resultados entre os intangíveis e a dispersão das previsões, a variável AI mostrou-se associada à redução da incerteza gerada pelos intangíveis, uma vez que a dispersão, segundo Barron et al. (1998), é capaz de refletir tal incerteza.

Tal resultado vai de encontro a outras evidências empíricas sobre o tema e também ao apontado pela teoria, quando indica que apenas aqueles intangíveis que se aproximem mais dos tangíveis serão reconhecidos pela contabilidade como ativos intangíveis (Zanoteli, 2015). Anteriormente, Matolcsy e Wyatt (2006) evidenciaram que o maior reconhecimento dos intangíveis (variável AI, neste trabalho) está associado a lucros mais previsíveis, gerando um aumento da acurácia e redução da dispersão das previsões, enquanto Snirivasan (2007) evidenciou que o $\mathrm{P} \& \mathrm{D}$ possui um efeito positivo nas previsões. Contrariamente, outros estudos apontam que empresas com maior atividade de inovação são mais difíceis de avaliar, estando os intangíveis associados negativamente às previsões, indicando erros mais altos (menor acurácia) e maior dispersão das previsões. (Chalmers et al. 2012; Dinh et al. 2015; Jia, 2017)

Já o Goodwill apresentou, como esperado, associação negativa com a acurácia das previsões (AP) e associação positiva com a dispersão das previsões (DP) dos analistas financeiros. Dessa forma, considera-se o Goodwill uma informação mais complexa e incerta, uma vez que uma maior complexidade e incerteza dos intangíveis pode afetar negativamente a previsibilidade dos lucros futuros. Outros trabalhos sugerem que a dificuldade de avaliar os intangíveis acaba reduzindo a qualidade das previsões (Barron et al., 2002; Dinh et al., 2015; Gu \& Wang, 2005). Tal fato pode ser justificado pela discricionariedade do valor atribuído ao custo de aquisição de outra empresa.

Apesar de não terem sidos encontrados estudos que abordem o Goodwill neste contexto de relação com as previsões dos analistas, tais resultados, até mesmo por serem contrários aos encontrados em relação às variáveis AI e IPD analisados anteriormente, mostram-se compatíveis aos achados de Jia (2017), Chalmers et al. (2012) e Dinh et al. (2015), ao indicar que o intangível está associado negativamente às previsões e demonstrando menor acurácia e maior dispersão das previsões. Mas, em linhas gerais, os resultados do trabalho apresentaram a existência de efeitos distintos entre os intangíveis estudados, de modo que as resoluções se mostraram mais aproximadas aos achados de Maaloul et al. (2016) que, apesar de evidenciarem que o aumento do disclosure dos intangíveis impactam positivamente as previsões dos analistas, ressaltaram que este efeito pode variar conforme o tipo do intangível, como foi possível observar nesta pesquisa.

\section{CONCLUSÃO}

Este trabalho teve como objetivo geral investigar a relação entre os intangíveis e as previsões dos analistas financeiros, tendo em vista que a expectativa de desempenho futuro dos intangíveis deve ser incorporada pelos analistas financeiros. Especificamente, buscou-se analisar se os intangíveis, mensurados pelo investimento em $\mathrm{P} \& \mathrm{D}$, ativo intangível reconhecido nas demonstrações financeiras, e o Goodwill exercem influência sobre a acurácia das previsões dos analistas financeiros, assim como na dispersão destas previsões. Para que o objetivo geral e os específicos fossem atingidos, foram utilizadas as seguintes proxies das previsões dos analistas: acurácia das previsões dos analistas financeiros, calculada pela mediana das previsões (AP), e dispersão das previsões dos analistas (DP). Já os intangíveis foram mensurados pelos investimentos com P\&D (IPD), ativos intangíveis reconhecidos nas demonstrações financeiras (AI) e o Goodwill (GW). Dessa forma, alternando as duas variáveis dependentes para previsões dos analistas financeiros e as três variáveis independentes para o intangível (investimentos com 
P\&D, ativos intangíveis reconhecidos nas demonstrações financeiras e o Goodwill) nos modelos especificados, foram investigados os resultados de seis regressões econométricas.

Diante dos resultados, evidenciou-se a contribuição informacional dos investimentos em $\mathrm{P} \& \mathrm{D}$ e dos ativos intangíveis reconhecidos nas demonstrações financeiras para a previsibilidade dos lucros futuros pelos analistas, aumentando a acurácia e reduzindo a dispersão das previsões financeiras. Os achados indicam que tais intangíveis fornecem informações que contribuem para tornar os lucros futuros menos incertos e mais previsíveis. Aprofundando os resultados, foi possível notar que o Goodwill contribui para a redução da previsibilidade dos lucros, o que pode ser explicado pela discricionariedade existente no processo de aquisição de outras empresas, o que nem sempre é amplamente divulgado.

Em linhas gerais, foi possível responder ao problema de pesquisa ao evidenciar que os intangíveis estão associados às previsões de lucros dos analistas financeiros. As evidências encontradas sugerem que os intangíveis estão associados à acurácia das previsões, confirmando a hipótese de pesquisa H1 e, com a dispersão das previsões, confirmando a hipótese de pesquisa H2. Sendo tais resultados relacionados ao ativo intangível (AI) e P\&D (IPD) e compatíveis com os achados de Matolcsy e Wyatt (2006) e Snirivasan (2007), corroborando as evidências existentes no sentido de que, em média, o reconhecimento de ativos intangíveis está associado a lucros mais previsíveis. Todavia, os resultados são contrários aos observados por Dinh et al. (2015), Jia (2017) e Chalmers et al. (2012), que apontaram que a capitalização dos custos de desenvolvimento está significativamente associada, tanto a maiores erros de previsão dos analistas quanto à maior dispersão da previsão. Observou-se a existência de relação estatística significante entre os intangíveis e as previsões dos analistas financeiros.

Entretanto, os resultados são significativos e contrários quando analisamos o intangível de Goodwill, evidenciando que tal relação pode ser positiva ou negativa, dependendo da complexidade e incerteza atrelada à avaliação do intangível em questão, apontando melhora na acurácia das previsões quando analisados os investimentos em P\&D e ativos intangíveis reconhecidos nas demonstrações financeiras, mas piora quando analisados os intangíveis de Goodwill. Os achados desta pesquisa sugerem que o impacto dos intangíveis sobre a previsão dos analistas financeiros não pode ser generalizado, pois o impacto ocorre de forma diferenciada conforme o intangível analisado. E, assim como Maaloul et al. (2016), deve-se ressaltar que o sinal desta associação pode variar em função do tipo de intangível estudado e seu grau de complexidade e incerteza.

Este trabalho contribui por preencher uma lacuna existente na discussão sobre os efeitos dos intangíveis nas previsões dos analistas financeiros ao, além de investigar os efeitos sobre variáveis já discutidas na literatura, como o investimento em $\mathrm{P} \& \mathrm{D}$ e o ativo intangível reconhecido nas demonstrações financeiras, adicionar a relação do Goodwill sobre a acurácia e dispersão das previsões dos analistas financeiros. Além disso, por evidenciar resultados distintos entre os intangíveis, indicando a complexidade e incerteza dos intangíveis como uma possível causa para resultados distintos encontrados na literatura.

Contudo, algumas limitações desta pesquisa devem ser consideradas. Primeiramente, a amostra é composta por empresas listadas nos Estados Unidos, não sendo possível a generalização dos resultados obtidos. Com relação aos modelos utilizados, é preciso destacar que os estes não consideram as influências de outros fatores sobre as previsões dos analistas financeiros. Sugerese, para pesquisas futuras, a utilização de outras proxies de intangível para verificar o comportamento das previsões dos analistas, segregação das empresas pelo grau de intangibilidade e a investigação desta relação em diferentes contextos, os quais podem influenciar os níveis de incerteza dos intangíveis, como em diferentes países e setores econômicos. 


\section{REFERÊNCIAS}

Abeysekera, I. (2016). Does the classification of intangibles matter? An equivalence testing. Advances in accounting, 35, 135-142.

Abhayawansa, S., \& Guthrie, J. (2016). Does intellectual capital disclosure in analysts' reports vary by firm characteristics? Advances in accounting, 35, 26-38.

Ali, A., Ciftci, M., \& Cready, W. M. (2012). Market underestimation of the implications of R\&D increases for future earnings: the US evidence. Journal of Business Finance \& Accounting, 39(3-4), 289-314.

Almeida, J. E. F., \& Dalmácio, F. Z. (2015). The effects of corporate governance and product market competition on analysts' forecasts: evidence from the Brazilian capital market. The International Journal of Accounting, 50(3), 316-339.

Amir, E., Lev, B., \& Sougiannis, T. (2003). Do financial analysts get intangibles? European Accounting Review, 12(4), 635-659.

Bae, J., Hur, W., Lee, J., \& Goh, J. (2017). Patent citations and financial analysts' long-term growth forecasts. Sustainability, 9(5), 846.

Barron, O. E., Kim, O., Lim, S. C., \& Stevens, D. E. (1998). Using analysts' forecasts to measure properties of analysts' information environment. Accounting Review, 421-433.

Barron, O. E., Byard, D., Kile, C., \& Riedl, E. J. (2002). High-technology intangibles and analysts' forecasts. Journal of Accounting Research, 40(2), 289-312.

Barth, M. E., Kasznik, R., \& McNichols, M. F. (2001). Analyst coverage and intangible assets. Journal of Accounting Research, 39(1), 1-34.

Burgman, R., \& Roos, G. (2004). The new economy: a new paradigm for managing for shareholder value. IC Congress, Centre for Business Performance, Cranfield, Reino Unido.

Chakravarty, A., \& Grewal, R. (2016). Analyst earning forecasts and advertising and R\&D budgets: role of agency theoretic monitoring and bonding costs. Journal of Marketing Research, 53(4), 580-596.

Cummins, J. G. (2005). A new approach to the valuation of intangible capital. In Corrado, C., Haltiwanger J., \& Sichel, D. Measuring capital in the new economy (pp. 47-72). Chicago: University of Chicago Press.

Currim, I. S., Lim, J., \& Zhang, Y. (2018). Effect of analysts' earnings pressure on marketing spending and stock market performance. Journal of the Academy of Marketing Science, 46(3), 431-452.

Dalmácio, F. Z. (2009). Mecanismos de governança e acurácia das previsões dos analistas do mercado brasileiro: uma análise sob a perspectiva da teoria de sinalização. Tese de doutorado, Universidade de São Paulo, São Paulo, SP, Brasil.

Damodaran, A. (2007). Dealing with intangibles: valuing brand names, flexibility and patents. Flexibility and Patents (April 7, 2007). doi: 10.2139/ssrn.1374562 
Diamond, D. W., \& Verrecchia, R. E. (1991). Disclosure, liquidity, and the cost of capital. The Journal of Finance, 46(4), 1325-1359.

Dinh, T., Eierle, B., Schultze, W., \& Steeger, L. (2015). Research and development, uncertainty, and analysts' forecasts: the case of IAS 38. Journal of International Financial Management \& Accounting, 26(3), 257-293.

Fávero, L. P., \& Belfiore, P. (2014). Métodos Quantitativos com Stata: procedimentos, rotinas e análise de resultados (Vol. 1). Amsterdam: Elsevier.

Fusai, G., \& Roncoroni, A. (2007). Implementing models in quantitative finance: methods and cases. Berlin: Springer Science \& Business Media.

Gentry, R. J., \& Shen, W. (2013). The impacts of performance relative to analyst forecasts and analyst coverage on firm R\&D intensity. Strategic Management Journal, 34(1), 121-130.

Gu, F., \& Wang, W. (2005). Intangible assets, information complexity, and analysts' earnings forecasts. Journal of Business Finance \& Accounting, 32(9-10), 1673-1702.

Iudicibus, S. (2010). Teoria da Contabilidade (10a ed.) São Paulo: Atlas.

Jia, N. (2017). Corporate Innovation Strategy, Analyst Forecasting Activities and the Economic Consequences. Journal of Business Finance \& Accounting, 44(5-6), 812-853.

Kanodia, C., Sapra, H., \& Venugopalan, R. (2004). Should Intangibles Be Measured: what are the economic trade-offs? Journal of Accounting Research, 42(1), 89-120.

Kothari, S. P. (2001). Capital markets research in accounting. Journal of Accounting and Economics, 31(1), 105-231.

Kwon, S. S. (2002). Financial analysts' forecast accuracy and dispersion: high-tech versus lowtech stocks. Review of Quantitative Finance and Accounting, 19(1), 65-91.

Lang, M. H., \& Lundholm, R. J. (1996). Corporate disclosure policy and analyst behavior. Accounting review, 467-492.

Lev, B. (2001). Intangibles: management, measurement, and reporting. Washington, DC: Brookings Institution Press.

Lev, B. (2005). Intangible assets: concepts and measurements. Encyclopedia of social measurement, 2(1), 299-305.

Luo, X., Homburg, C., \& Wieseke, J. (2010). Customer satisfaction, analyst stock recommendations, and firm value. Journal of Marketing Research, 47(6), 1041-1058.

Maaloul, A., Ben Amar, W., \& Zeghal, D. (2016). Voluntary disclosure of intangibles and analysts' earnings forecasts and recommendations. Journal of Applied Accounting Research, 17(4), 421-439. 
Martins, E., Diniz, J. A., \& Miranda, G. J. (2012). Análise avançada das demonstrações contábeis: uma abordagem crítica. São Paulo: Atlas.

Matolcsy, Z., \& Wyatt, A. (2006). Capitalized intangibles and financial analysts. Accounting \& Finance, 46(3), 457-479.

McDermott, C. M. (1999). Managing radical product development in large manufacturing firms: a longitudinal study. Journal of Operations Management, 17(6), 631-644.

Palmon, D., \& Yezegel, A. (2012). R\&D intensity and the value of analysts' recommendations. Contemporary Accounting Research, 29(2), 621-654.

Penman, S. H. (2009). Accounting for intangible assets: there is also an income statement. Abacus, 45(3), 358-371.

Perez, M. M., \& Famá, R. (2006). Ativos intangíveis e o desempenho empresarial. Revista Contabilidade \& Finanças, 17(40), 7-24.

Santos, J. L. D., Gomes, J. M. M., Fernandes, L. A., Pinheiro, P. R., \& Schmidt, P. (2006). Ativos intangíveis: fonte de vantagem competitiva. Contexto, 6(10), 29-46.

Simões, J. J. F., \& Louzada, L. C. (2016). O Intangível Como Fator De Vantagem Competitiva. Congresso Nacional de Administração e Contabilidade - AdCont, Rio de Janeiro, RJ, 17.

Scott, W. R. (2012). Financial accounting theory (6a ed.) Toronto: Pearson Prentice Hall.

Srinivasan, R. (2007). Do Analysts Pay Attention to Marketing? Advertising, R\&D, and Dispersion in Analysts' Forecasts. Red McCombs School of Business, University of Texas, Austin, TX, Estados Unidos.

Zanoteli, E. J. (2015). Ativos intangíveis: uma perspectiva teórica para o seu reconhecimento e mensuração nas demonstrações contábeis. Tese de doutorado em Administração, Universidade Federal de Minas Gerais, Belo Horizonte, MG, Brasil. 